\title{
Avaliação da qualidade de água do rio Pardo (MG) com base em parâmetros físicos, químicos e microbiológicos
}

\author{
Evaluation of the water quality of the Pardo River (MG) based on physical, chemical and \\ microbiological parameters \\ Evaluación de la calidad del agua del río Pardo (MG) en base a parámetros físicos, químicos y \\ microbiológicos
}

Recebido: 27/04/2021 | Revisado: 02/05/2021 | Aceito: 06/05/2021 | Publicado: 07/05/2021

\author{
Maria Fernanda Antunes Collares \\ ORCID: https://orcid.org/0000-0003-3111-5380 \\ Universidade Federal de Viçosa, Brasil \\ E-mail: maria.collares@ufv.br \\ Leonardo França da Silva \\ ORCID: https://orcid.org/0000-0002-9710-8100 \\ Universidade Federal de Viçosa, Brasil \\ E-mail: leonardo.silva@ufv.br \\ Rubens Barrichello Gomes Barbosa \\ ORCID: https://orcid.org/0000-0002-6806-0590 \\ Universidade Federal de Viçosa, Brasil \\ E-mailrubens.barbosa@ufv.br \\ Ana Carolina Chaves Dourado \\ ORCID: https://orcid.org/0000-0002-1106-1349 \\ Universidade Federal de Viçosa, Brasil \\ E-mail: ana.dourado@ufv.br \\ Bruna Nogueira Rezende \\ ORCID: https://orcid.org/0000-0003-4337-9324 \\ Universidade de São Paulo, Brasil \\ E-mail: bbrunarezende@ hotmail.com \\ João Antônio Costa do Nascimento \\ ORCID: https://orcid.org/0000-0001-5984-5997 \\ Universidade Federal de Viçosa, Brasil \\ E-mail: joao.nascimento@ufv.br
}

\begin{abstract}
Resumo
Este artigo apresenta uma análise do monitoramento do índice de qualidade da água e o emprego de técnicas estatísticas multivariadas na porção mineira do rio Pardo, visando selecionar os parâmetros mais significativos nos aspectos atuais da qualidade da água, agrupando as estações de acordo com à semelhança dos parâmetros estudados. Os dados utilizados no estudo, foram obtidos no Instituto Mineiro de Gestão de Águas - IGAM referentes aos meses de janeiro a outubro do ano de 2018. O índice de qualidade da água foi calculado para os 5 pontos de monitoramento e classificados de acordo com o IQA-NSF. Análise de componentes principais (ACP) e a análise de Cluster (AC) foram usadas para reduzir o número de variáveis e para agrupar estações com características semelhantes, respectivamente. A estação PD005 apresentou a menor média do índice de qualidade de água, isso se deve pelo fato do parâmetro de coliformes fecais se destacar negativamente em grande quantidade na estação. Usando o ACP, dois componentes principais foram selecionados como indicadores da qualidade da água explicando a variância cumulativa de $78 \%$. A AC agrupou as estações em três grupos podendo identificar a estação mais poluída e a estação menos poluída. Os resultados obtidos através de estatística multivariada provaram ser importantes para a compreensão da situação atual da qualidade da água na bacia e pode ser usado para melhorar a gestão dos recursos hídricos porque a coleta e análise de todos os parâmetros em todas as estações de monitoramento exigem maior disponibilidade recursos financeiros.

Palavras-chave: Rede de monitoramento; Bacia hidrográfica; Estatística multivariada; Análise de cluster.
\end{abstract}

\begin{abstract}
This article presents an analysis of the monitoring of the water quality index and the use of multivariate statistical techniques in the mining portion of the Pardo River, in order to select the most significant parameters in the current aspects of water quality, grouping the stations according to the similarity of the studied parameters. The data used in the study were obtained from the Minas Gerais Water Management Institute - IGAM for the months of January to October of the year 2018. The water quality index was calculated for the 5 monitoring points and classified according to the IQA -NSF. Principal component analysis (PCA) and Cluster analysis (CA) were used to reduce the number of
\end{abstract}


variables and to group stations with similar characteristics, respectively. The PD005 station presented the lowest average of the water quality index, this is due to the fact that the parameter of faecal coliforms stands out negatively in great quantity in the station. Using the PCA, two main components were selected as indicators of water quality explaining the cumulative variance of $78 \%$. The CA grouped the stations into three groups, being able to identify the most polluted and the least polluted stations. The results obtained through multivariate statistics have proved to be important for understanding the current water quality situation in the basin and can be used to improve the management of water resources because the collection and analysis of all parameters at all monitoring stations requires greater availability of financial resources.

Keywords: Monitoring network; Hydrographic basin; Multivariate statistics; Cluster analysis.

\section{Resumen}

Este artículo presenta un análisis del seguimiento del índice de calidad del agua y el uso de técnicas estadísticas multivariadas en el tramo minero del río Pardo, con el fin de seleccionar los parámetros más significativos en los aspectos actuales de la calidad del agua, agrupando las estaciones según la similitud de los parámetros estudiados. Los datos utilizados en el estudio se obtuvieron del Instituto de Gestión del Agua de Minas Gerais - IGAM para los meses de enero a octubre del año 2018. El índice de calidad del agua se calculó para los 5 puntos de monitoreo y se clasificó según el IQA -NSF. El análisis de componentes principales (ACP) y el análisis de conglomerados (AC) se utilizaron para reducir el número de variables y agrupar estaciones con características similares, respectivamente. La estación PD005 presentó el promedio más bajo del índice de calidad del agua, esto se debe a que el parámetro de coliformes fecales se destaca negativamente en gran cantidad en la estación. Utilizando el ACP, se seleccionaron dos componentes principales como indicadores de la calidad del agua que explican la varianza acumulada del $78 \%$. La CA agrupó las estaciones en tres grupos, pudiendo identificar las estaciones más contaminadas y las menos contaminadas. Los resultados obtenidos a través de estadísticas multivariadas han demostrado ser importantes para comprender la situación actual de la calidad del agua en la cuenca y pueden utilizarse para mejorar la gestión de los recursos hídricos porque la recopilación y análisis de todos los parámetros en todas las estaciones de monitoreo requiere una mayor disponibilidad de recursos financieros.

Palabras clave: Red de monitoreo; Cuenca hidrográfica; Estadística multivariante; Análisis de conglomerados.

\section{Introdução}

A água é um importante recurso para a sobrevivência humana e, dentre seus diversos usos se destaca o abastecimento humano e industrial, a irrigação, a produção de energia elétrica e as atividades de lazer e recreação (Oliveira, Santos \& Lima, 2017). s águas superficiais estão altamente expostas à poluição, descarga de águas residuais e são mais vulneráveis à contaminação, resultando em um declínio geral em qualidade (Anteneh et al., 2018).

A bacia hidrográfica do rio Pardo está localizada nos estados de Minas Gerais e da Bahia. A área de uso e ocupação do solo da bacia tem como principal ocupação econômica a pecuária, agricultura, extrativismo vegetal e mineração (HIDROTEC). A bacia hidrográfica do rio Pardo vem sofrendo com a poluição dos corpos hídricos, devido ao crescimento populacional no entorno da bacia e o uso e ocupação do solo feita de maneira desordenada.

A avaliação e monitoramento da qualidade da água é um dos aspectos mais importantes para garantir uma boa saúde ambiental. O Índice de Qualidade da Água (IQA) é uma das ferramentas mais frequentemente usadas para avaliar a qualidade geral dos recursos hídricos. Vários métodos e modelos estatísticos têm sido utilizados para a avaliação da qualidade e quantidade das águas, dentre eles se destaca a aplicação de técnicas estatísticas multivariadas que ajudam a identificar possíveis fatores e/ou fontes que influenciam os sistemas hídricos e oferecem uma ferramenta robusta para o gerenciamento confiável dos recursos hídricos, bem como uma solução rápida para problemas de poluição em muitas partes do mundo (Bodrud-Doza et al., 2016).

Tais métodos estatísticos são apropriados para lidar com parâmetros múltiplos e variáveis que, quando combinados, podem contribuir para a avaliação da qualidade da água (Li et al., 2018). A análise de clusters permite classificar as unidades de acordo com um critério de seleção predeterminado resultante de alta homogeneidade interna (dentro do cluster) e alta heterogeneidade externa (entre os clusters) (Muangthong \& Shrestha, 2015).

Portanto, a análise e o monitoramento da qualidade da água são muito importantes para a definição de tecnologias e medidas a serem tomadas em seu tratamento. Nesse sentido, o objetivo do presente estudo consiste em calcular e avaliar o 
índice de qualidade de água da bacia hidrográfica do rio Pardo por meio de parâmetros físico, químicos e da aplicação da estatística multivariada.

\section{Metodologia}

Apresentando uma área de drenagem de $32.050 \mathrm{~km}^{2}$, com população estimada em mais de 260 mil habitantes, a bacia hidrográfica do rio Pardo, área de estudo do presente trabalho (Figura 1) abrange quase 30 municípios com extensas áreas rurais praticantes de agropecuária. Além disso, a área de estudo possui como principal ocupação econômica a pecuária, agricultura, extrativismo vegetal e mineração (HIDROTEC).

Figura 1. Localização geográfica da Bacia Hidrográfica do Rio Pardo.

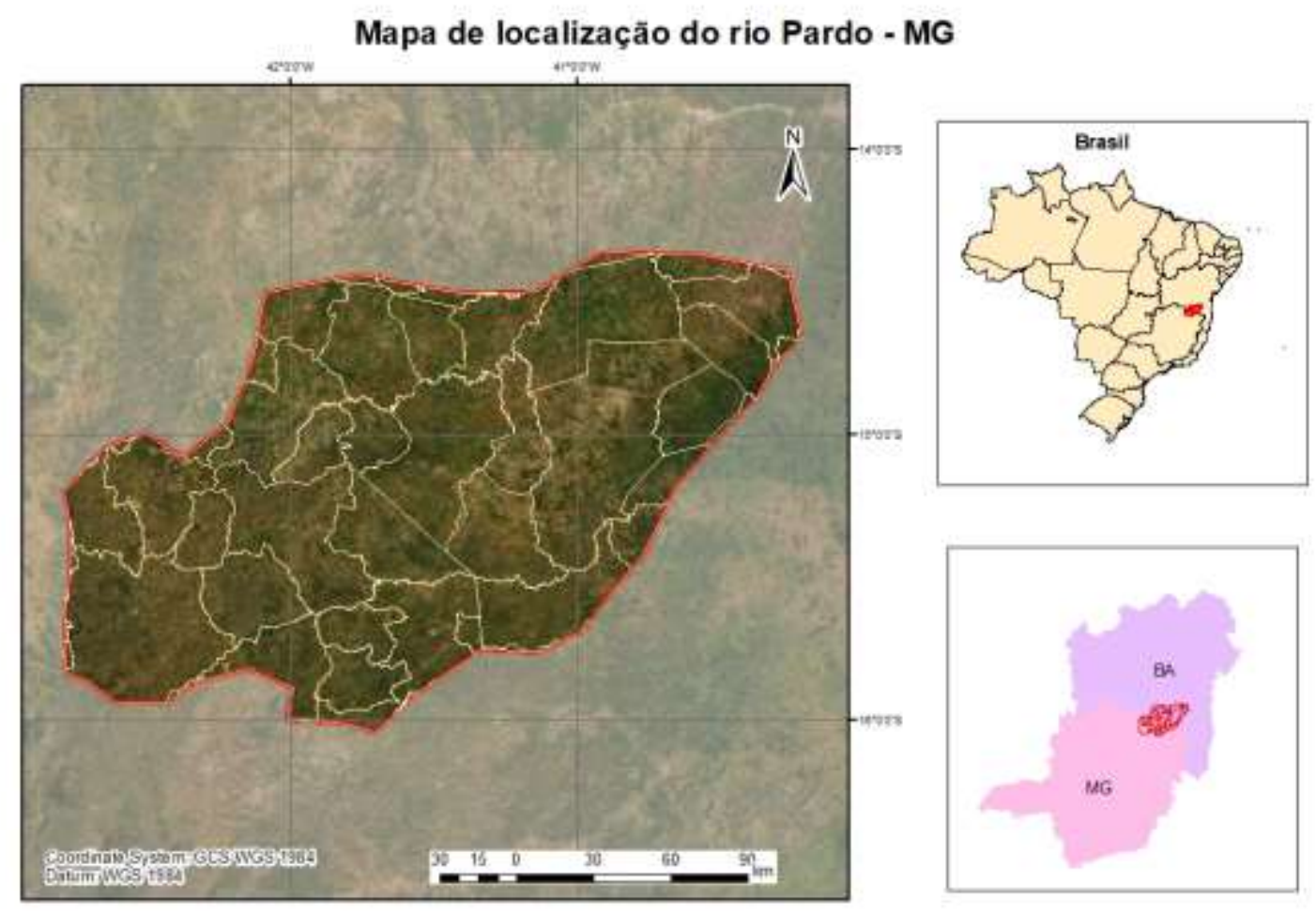

Fonte: Autores.

De acordo com o programa HIDROTEC a área de drenagem do rio Pardo em território mineiro abrange $12.728 \mathrm{~km}^{2} \mathrm{e}$ ocupa o nono lugar em termos de produção de água e contribui com 0,2\% da vazão mínima produzida no Estado.

\subsection{Estações de monitoramento}

Os dados de monitoramento da qualidade de água utilizados na realização deste trabalho foram obtidos junto ao Instituto Mineiro de Gestão de Águas - IGAM referentes aos meses de janeiro a outubro do ano de 2018. Segundo o IGAM, o curso hídrico é enquadrado como classe 2. Foram analisadas 5 estações de monitoramento (PD002, PD003, PD005, PD006 e PD007) localizadas ao longo do trecho do rio Pardo, sendo estas nomeadas de P1, P2, P3, P4 e P5, respectivamente.

Os vetores do limite da bacia estudada, dos rios e das sedes municipais foram obtidos na Agência Nacional das Águas - ANA. Na Tabela 1 apresenta características das 5 estações de monitoramento da bacia hidrográfica do rio Pardo, no qual estão localizadas os corpos hídricos, a descrição, a nomenclatura adotada no trabalho e os municípios que estão inseridas. 
Tabela 1. Estações de monitoramento da qualidade da água do rio Pardo.

\begin{tabular}{c|cccc}
\multicolumn{1}{c}{ Estação } & Nomenclatura & $\begin{array}{c}\text { Cursos } \\
\text { hídricos }\end{array}$ & Descrição & Município \\
\hline PD002 & P1 & $\begin{array}{c}\text { Rio do } \\
\text { Cedro }\end{array}$ & $\begin{array}{c}\text { Rio do Cedro, a jusante da cidade } \\
\text { de Santo Antônio do Retiro. }\end{array}$ & $\begin{array}{c}\text { Santo Antônio do } \\
\text { Retiro-MG }\end{array}$ \\
PD003 & P2 & $\begin{array}{c}\text { Rio Pardo } \\
\text { (PA1) }\end{array}$ & $\begin{array}{c}\text { Rio Pardo a jusante da cidade de } \\
\text { Rio Pardo de Minas }\end{array}$ & Indaiabira-MG \\
PD005 & P3 & $\begin{array}{c}\text { Rio Pardo } \\
\text { (PA1) }\end{array}$ & $\begin{array}{r}\text { Rio Pardo na cidade de Candido } \\
\text { Sales / BA }\end{array}$ & Cândido Sales-BA \\
PD006 & P4 & $\begin{array}{c}\text { Rio Pardo } \\
\text { (PA1) }\end{array}$ & $\begin{array}{c}\text { Rio Pardo a Jusante de Machado } \\
\text { Mineiro - Coleta de balsa }\end{array}$ & Ninheira-MG \\
PD007 & P5 & $\begin{array}{c}\text { Rio São } \\
\text { João do } \\
\text { Paraíso }\end{array}$ & $\begin{array}{c}\text { Rio São João do Paraíso no } \\
\text { município de São João do Paraíso. }\end{array}$ & São João do Paraíso - \\
MG
\end{tabular}

Fonte: IGAM (2018).

\subsection{Avaliação dos parâmetros de qualidade da água}

Dentre as diversas ferramentas que determinam a qualidade da água baseadas em características físicas, químicas e biológicas da água, o Índice de Qualidade da Água (IQA) é uma metodologia de suma importância para determinar os padrões das águas nacionais através dos pesos dos parâmetros adequados as características regionais (ANA, 2017). A partir dos dados mensais obtidos para o ano de 2018, realizou-se uma análise comparativa, correlacionando os resultados com os limites máximos estabelecidos para os corpos d'água de classe 2 , já estabelecido pelos dados do IGAM, pela resolução CONAMA $n^{\circ}$ 357/2005 (CONAMA,2005).

\subsection{Cálculo do Índice de Qualidade de Água - IQA}

A National Sanitation Foundation (NSF) criou o índice de qualidade de água (IQA) com objetivo de comparar e monitorar a qualidade dos corpos hídricos analisando a contaminação por esgotos domésticos, industriais e agropecuários (LOPES et al., 2008). Portanto, o IQA criado pela NSF avalia os parâmetros oxigênio dissolvido, coliformes termotolerantes, $\mathrm{pH}$, demanda bioquímica de oxigênio, nitrato, fósforo total, temperatura, turbidez e sólidos totais e possui variação de zero (pior qualidade) a 100 (melhor qualidade) com seus respectivos pesos (Wi) (Castro Junior, Sobreira \& Bortoloti, 2007).

O monitoramento da qualidade da água através do IQA traz informações de maneira clara para a população através dos indicadores que compõe o índice (Maia, Silva \& Libânio, 2019). Os dados foram coletados nos meses de janeiro, abril, agosto e outubro sendo 5 pontos analisados em cada mês nas respectivas cidades: Santo Antônio do Retiro- MG, IndaiabiraMG, Cândido Sales- BA, Ninheira- MG e São João do Paraíso - MG (Tabela 2). Para cada parâmetro observado foi atribuído um peso (w) de acordo com von Sperling (2007). 
Tabela 2. Parâmetros de qualidade da água.

\begin{tabular}{c|ccc}
\multicolumn{1}{c}{ Parâmetro } & Nomenclatura & Unidade & Peso (w) \\
\hline Coliformes & Coli & NMP $/ 100 \mathrm{~mL}$ & 0,15 \\
termotolerantes & & & \\
$p H$ & $\mathrm{pH}$ & - & 0,12 \\
DBO ${ }_{5}^{20}$ & $\mathrm{DBO}$ & $\mathrm{mg} / \mathrm{L}$ & 0,10 \\
Nitrogênio Total & $\mathrm{NT}$ & $\mathrm{mgN} / \mathrm{L}$ & 0,10 \\
Fósforo Total & $\mathrm{PT}$ & $\mathrm{mgP} / \mathrm{L}$ & 0,10 \\
Diferença de & $\mathrm{DifT}$ & ${ }^{\circ} \mathrm{C}$ & 0,10 \\
Temperatura & & & \\
Turbidez & $\mathrm{Turb}$ & $\mathrm{NTU}$ & 0,08 \\
Sólidos Totais & $\mathrm{ST}$ & $\mathrm{mg} / \mathrm{L}$ & 0,08 \\
OD & $\mathrm{OD}$ & $\%$ satur & 0,17 \\
\hline
\end{tabular}

Fonte: IQA-NSF.

O Índice de Qualidade de Água foi calculado pela Equação 1 de acordo com a NSF (2007):

$I Q A=\prod_{i=1}^{n} q i^{w i}$

Os parâmetros estudados foram agrupados de modo que possibilitem a classificação qualitativa das águas segundo IQA-NSF apresentado na Tabela 3. Em modelos empíricos os pesos de cada atributo variam de acordo com a região devido a fatores climáticos, condições geológicas e usos da terra

Tabela 3. Classificação da qualidade da água segundo IQA-NSF.

\begin{tabular}{c|c} 
Índice (IQA) & Qualidade \\
\hline $90<I Q A \leq 100$ & Excelente \\
$70<I Q A \leq 90$ & Bom \\
$50<I Q A \leq 70$ & Média \\
$25<I Q A \leq 50$ & Ruim \\
$0<I Q A \leq 25$ & Muito Ruim \\
\hline
\end{tabular}

Fonte: IQA-NSF.

\subsection{Aplicação da estatística multivariada}

A análise de cluster hierárquica foi usada para testar a heterogeneidade espacial nas concentrações de constituintes entre as estações de monitoramento da bacia do rio Pardo. Para cada estação, a variável de qualidade da água foi padronizada em escala $Z$ para ter uma média de 0 e desvio padrão de 1, para garantir a mesma escala para todos os parâmetros de qualidade da água (LIU et al.,2018).

A AC foi calculada por meio do método de Ward, usando a distância euclidiana quadrada como uma medida de similaridade (Muangthong \& Shrestha, 2015). Muangthong \& Shrestha (2015) afirmam que o método de Ward utiliza uma abordagem de análise de variância para avaliar a distância entre os clusters.

Após o cálculo da análise de cluster, calculou-se a análise de componentes principais (ACP) para avaliar a relação obtida entre as variáveis, mostrando a atuação dos elementos do IQA na qualidade da água (Cecconello, Centeno \& Guedes, 
2018). Além disso, ACP auxiliou na obtenção dos componentes principais (CP) que interferem no cálculo do IQA das estações de monitoramento da bacia hidrográfica do rio Pardo. A AC e a ACP foram calculadas utilizando o software Rstudio.

\section{Resultados e Discussão}

\subsection{Avaliação dos parâmetros de qualidade da água}

Os dados referentes aos parâmetros físicos, químicos e biológicos obtidos das análises das amostras de água do rio Pardo, coletados nos pontos P1, P2, P3, P4 e P5, nos meses de janeiro, abril, agosto e outubro de 2018, estão apresentados na Tabela 4.

Tabela 4. Valores dos parâmetros físicos, químicos e biológicos das amostras de água do rio Pardo coletadas para o período avaliado (janeiro, abril, agosto e outubro de 2018).

\begin{tabular}{|c|c|c|c|c|c|c|c|c|c|c|c|c|c|c|c|c|c|c|c|c|}
\hline \multirow[t]{2}{*}{ Perímetros } & \multicolumn{5}{|c|}{ JANEIRO } & \multicolumn{5}{|c|}{ ABRIL } & \multicolumn{5}{|c|}{ ACosto } & \multicolumn{5}{|c|}{ OtTLRO } \\
\hline & p1 & $\mathbf{P 2}$ & P3 & P4 & P5 & $\mathbf{p 1}$ & $P_{2}$ & P3 & P4 & PS & P1 & P2 & P3 & P4 & P5 & PI & $\mathbf{p}_{2}$ & P3 & P4 & P5 \\
\hline Terperanura & 25,10 & 25,70 & 28,20 & 27,80 & 25,50 & 23,30 & 28,70 & 24,00 & 25.00 & 21,40 & 18.60 & 21,10 & 28.60 & 23,90 & 17,90 & 23,60 & 24,60 & 25.30 & 25,90 & 22,20 \\
\hline Tarbidez & 17,30 & 19.20 & 62,60 & 21.10 & $11, \infty 0$ & $\$ 2,20$ & 10,30 & 6,12 & 29,20 & 23,20 & 6,74 & 7,13 & 7.44 & 20,30 & 5,30 & 17,50 & 11,00 & 5,87 & 12,30 & $17, \infty 0$ \\
\hline Conciumsidato & 301000 & 341.00 & 250,00 & 245,00 & $P, 10$ & 22,20 & 20,20 & 28,00 & it, 80 & 94,80 & s.:70 & 53,20 & sa,so & 10,30 & 32,10 & 48,70 & 41,30 & 45,50 & 75.30 & 19.50 \\
\hline$p h$ & 0,50 & 9,80 & 9,70 & 6.50 & 0,00 & 5.60 & 590 & 2,50 & 6.50 & 5.00 & 0.25 & 60 & 7,10 & 7,10 & 0.30 & 9,90 & 0,40 & 5,60 & 0.20 & 3,00 \\
\hline$O D$ & $3, \pi$ & 4,70 & 3,90 & 5,90 & 2,20 & 6,50 & 6.40 & 2,70 & 4,40 & 3,10 & 2,80 & 7,10 & 3,20 & 6,30 & 5,00 & 2,70 & 6,30 & 2,60 & 5.40 & 6,10 \\
\hline Nitriso & 0,35 & 0,90 & 0.57 & 0,47 & 0,51 & 0,10 & a.20 & 0,58 & 0,52 & 0,11 & 0.10 & a,10 & 0,42 & $0,0.8$ & 0,10 & 0,10 & 0,12 & 0,05 & a.to & 0,11 \\
\hline Fodyoro & $0, \infty 6$ & $a, a 7$ & 0,08 & 0,20 & 0,07 & 0,02 & 0,02 & 0,05 & 0,02 & 0,03 & 0.02 & $a, 02$ & 0.00 & 0.92 & 0.02 & 0,04 & 0.02 & 0,07 & 0.02 & 0,0, \\
\hline E. Coit & 1485,00 & 41,30 & 1954,80 & 1015,20 & 225,50 & 487,40 & 62,90 & 599,20 & 23,10 & 499,00 & 322,00 & 41,30 & 291,70 & 932,10 & 215,7 & 148.00 & 30,40 & 2073,2 & 458,00 & 690.70 \\
\hline Coliformes & 2419,60 & 1955,90 & 2419,60 & 980,39 & 1732,89 & 2319,6 & 2172,1 & 2319,6 & 035,17 & 1046.24 & 1046,24 & 1020,9 & 2419,6 & 2755,4 & 2348 & $2419,6-$ & 2611,1 & 2419,6 & 2419,57 & $24: 9,6$ \\
\hline$D B O$ & $2, \infty 0$ & $2, \infty$ & 2,00 & $2, \infty 0$ & 2,00 & $2, \infty$ & 2000 & 3,00 & 2,00 & 2,00 & 2.00 & 200 & 4.70 & 2.00 & 2,00 & 2,20 & $2, \infty$ & $1, \infty$ & 2.00 & 2,00 \\
\hline$D Q O$ & 11.00 & $\$ 4.00$ & 25,00 & 20,00 & 10,09 & 14,00 & 14,00 & 18,00 & 29,90 & 2400 & 19.00 & 39,00 & 36,00 & 28,00 & 37,00 & 96,00 & 1000 & $2 n, 00$ & 15,00 & $19, \infty 0$ \\
\hline NT & 0.16 & 0.19 & 0.91 & a.15 & 0,10 & 0.10 & 0.10 & 0.15 & $0,2 \mathrm{n}$ & 0,10 & 0.31 & 0.20 & 0.21 & 0.10 & 0,10 & 0,4 & 0,10 & 0,10 & 0.10 & 0,10 \\
\hline ST & 58.00 & 59.00 & 232,00 & 100,00 & 80,00 & 47,00 & 50,00 & 192,00 & 35,00 & 34,00 & 28,00 & 96,00 & 29600 & 85,00 & 48,00 & 49.00 & 4900 & 176000 & 61,00 & 53,00 \\
\hline
\end{tabular}

Fonte: Autores.

De acordo com a Tabela 4, os valores de temperatura variaram na faixa de $17,9^{\circ} \mathrm{C}$ no ponto $\mathrm{P} 5$ do mês de agosto e $28,2{ }^{\circ} \mathrm{C}$ no P2 no mês de janeiro, indicando que o lançamento de efluentes no rio não apresentou impacto relevante sobre a temperatura da água e a maior temperatura em janeiro já era previsto devido ao período de verão.

Os resultados de turbidez em todos os pontos monitorados ficaram abaixo de 100 UNT, estando, assim, de acordo com os limites estabelecidos pela lei CONAMA n³57/2005, cujo padrão é de até 100UNT para corpos d'água de classe 2. Com isso, indica-se que os valores de turbidez não interferem de forma significativa nos processos fotossintéticos das plantas aquáticas e das algas do rio Pardo. O valor de turbidez mais alto na época de chuva pode ser explicado devido ao escoamento superficial que transporta sedimentos de terras expostas para o rio (Miyittah et al., 2020).

Os valores encontrados de $\mathrm{pH}$ variaram de 5,6 a 7,1 o que pode ser um limitante, já que os valores limites de $\mathrm{pH}$ para os sistemas aquáticos estabelecidos pela resolução é na faixa de pH 6,0 a 9,0. Algumas estações de monitoramento apresentaram valores de $\mathrm{pH}$ abaixo do limite da resolução indicando possível mortalidade de peixes no ambiente. Miyittah $e t$ al., (2020) em seu trabalho de avaliação da qualidade da água em lagoas encontraram resultados de $\mathrm{pH}$ semelhantes variando de 5,96 a 6,90 concluindo que o meio ácido pode afetar o sabor, a solubilidade de metal tóxico e a mortalidade dos peixes. Além disso, Miyittah et al., (2020) afirmam que pH com valores mais elevados podem estar associados ao aporte de hidróxidos, bicarbonatos e fosfatos associados ao desgaste natural das rochas e de fontes antropogênicas transportados por escoamento superficial para o rio.

O OD de acordo com a legislação não pode ser inferior a $5 \mathrm{mg} / \mathrm{L}$, e em todos os meses de monitoramento, algumas estações apresentaram valores abaixo do recomendado. Em janeiro, as estações P2, P3 e P5; em abril as estações P3, P4 e P5; em agosto P1 e P3 e em outubro as estações P1 e P3 apresentaram valores inferiores ao recomendado. Nota-se que todas as estações apresentaram uma irregularidade em alguma época do ano, o que se pode concluir que os valores estão associados a matéria orgânica e a decomposição dos resíduos (Miyittah et al., 2020) resultados também encontrados por Seu-Anoï et al. 
(2018). O maior valor de OD foi durante a época de seca podendo estar relacionado com as menores quantidades de escoamento que geralmente carregam nutrientes e resíduos para os corpos d'água. Além disso, Miyittah et al. (2020) afirmam que os baixos valores de OD podem sufocar algumas espécies de peixes, assim como causar desequilíbrio no ecossistema aquático.

A quantidade de fósforo no rio provém especialmente de resíduos municipais e escoamento da utilização de fertilizantes em excesso. Ademais, os níveis de fósforo sendo praticamente uniformes em todo o rio e nas estações de monitoramento podem ser uma indicação de diversas descargas pontuais e difusas de esgotamento doméstico, industriais e agrícolas (Miyittah et al., 2020).

Normalmente, a CE em águas superficiais é influenciada por íons dissolvidos de origem natural e antropogênica. CE mais alta na estação chuvosa é uma indicação provável de que quantidades significativas dos íons dissolvidos foram carreados para o rio por escoamento superficial da partir de fontes não pontuais, como terras agrícolas; uso dominante do solo na bacia (Miyittah et al., 2020).

Os dados referentes à determinação de nitrito revelam que a concentração varia na faixa de 0,01 a 0,95 mg.L-1, estando dentro do limite estabelecido pela resolução do CONAMA nº 357/2005 (CONAMA, 2005), que é de 1,0 mg.L-1. Este fato pode estar associado à reduzida ocorrência do processo de nitrificação e mesmo que, com relação a esse parâmetro, as águas do rio estão dentro dos padrões estabelecidos para corpos hídricos classe 2.

Foi verificado que todas as amostras de água analisadas possuem a presença de coliformes totais, cuja variação de concentração está na faixa de 435 a 2.700 UFC.100 mL-1 de amostra (Figura 2). Em geral, as águas coletadas no mês de outubro, apresentaram os maiores índices de coliformes totais, com contaminação de 2.400 a 2.600 UFC.100 mL-1, o que se deve, possivelmente, aos lançamentos de esgotos domésticos nas proximidades do referido local investigado. No entanto, a contaminação pode ser explicada pelo fato de o rio Pardo está inserido no perímetro urbano.

Figura 2. Presença de coliformes totais ao longo do período monitorado para as estações de monitoramento da bacia.

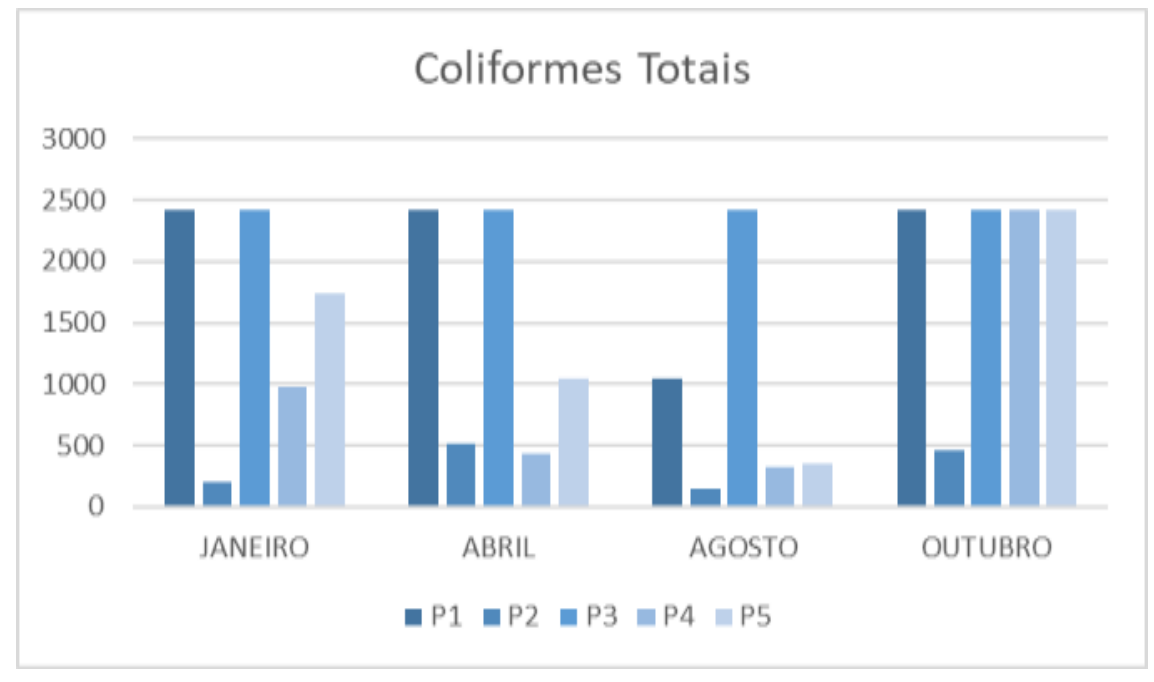

Fonte: Autores.

De acordo com as Resolução 357 do CONAMA, o rio Pardo tem seu enquadramento das águas aptas ao uso em recreações de contato secundário, porém inadequadas para a irrigação de hortaliças, de plantas frutíferas e de jardins, além de não serem aptas para uso na aquicultura e em atividades de pesca. Portanto, a alta contaminação por coliformes fecais revela o alto índice de deposição diária de resíduo orgânico animal no solo e/ou o lançamento de esgotos domésticos sem tratamentos 
prévios, de modo que aumente o risco da aquisição de doenças patogênicas por parte da população que entra em contato com as águas contaminadas.

\section{2 Índice de qualidade da água}

Os valores do índice da qualidade da água do rio Pardo de cada estação em cada mês de estudo estão apresentadas na Tabela 5 .

Tabela 5. Índice de qualidade da água do rio Pardo.

\begin{tabular}{c|ccccc} 
ESTAÇÕES & JANEIRO & ABRIL & AGOSTO & OUTUBRO & MÉDIA \\
\hline PI (PD002) & 99,24 & 96,72 & 67,73 & 64,89 & 82,14 \\
P2 (PD003) & 79,17 & 100,15 & 88,35 & 104,01 & 92,92 \\
P3 (PD005) & 76,17 & 67,89 & 71,98 & 68,11 & 71,03 \\
P4 (PD006) & 100,97 & 87,43 & 103,47 & 96,71 & 97,14 \\
P5 (PD007) & 64,24 & 66,24 & 90,79 & 94,93 & 79,05 \\
\hline
\end{tabular}

Fonte: Autores.

Analisando a Tabela 5, tem-se que a estação P3 apresentou a menor média do índice de qualidade de água, isso se deve ao fato do parâmetro de coliformes fecais se destacar em grande quantidade na estação. Além disso, a estação se encontra dentro da cidade de Cândido Sales, o que leva a concluir que o esgoto doméstico e as ações antrópicas estão influenciando nas características do rio.

Na Figura 3 apresenta as estações de monitoramento da qualidade da água com sua respectiva classificação levando em consideração a média do índice de qualidade da água do rio Pardo nos pontos monitorados.

Figura 3. Mapa do índice de qualidade da água do rio Pardo.

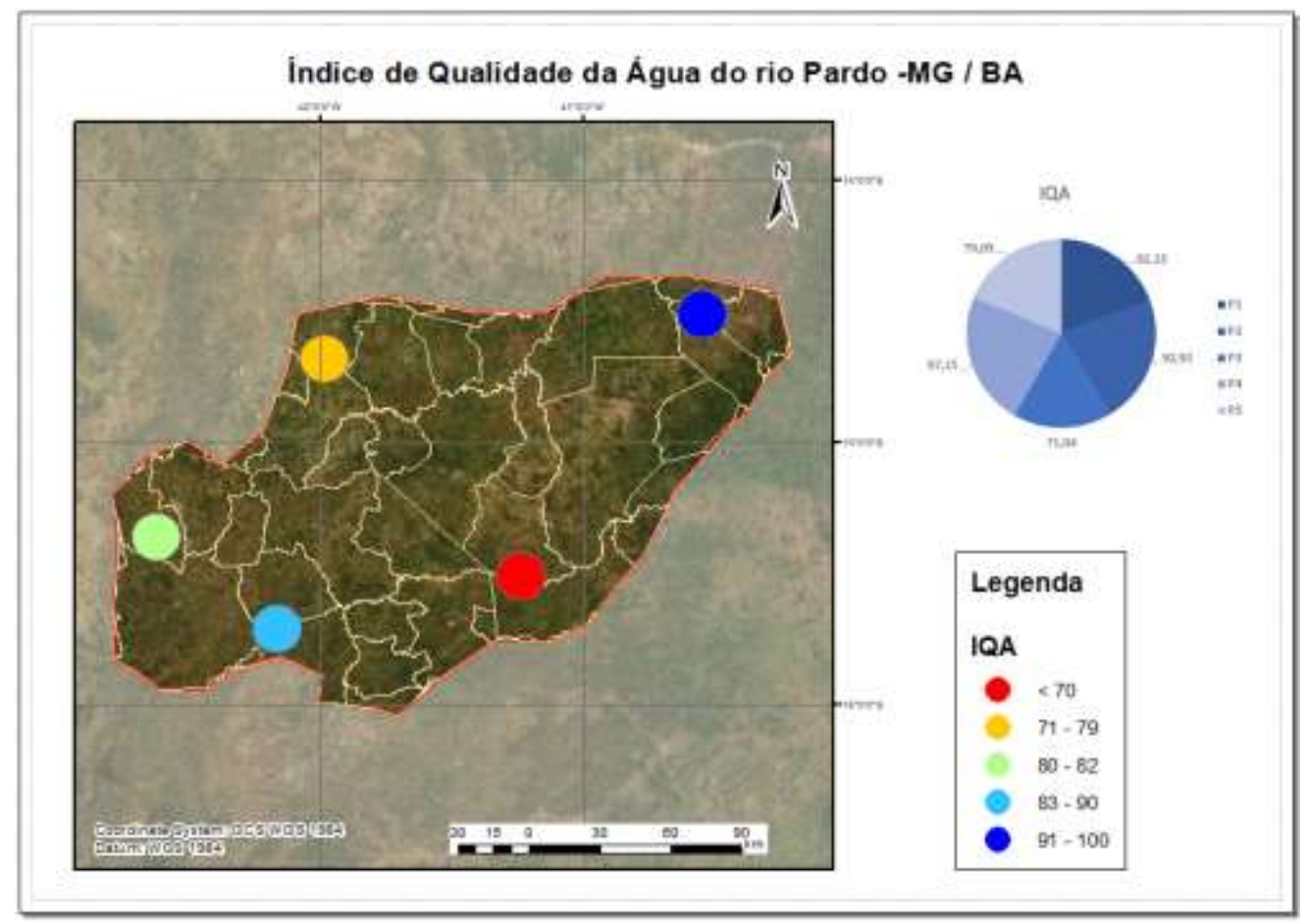

Fonte: Autores. 
Observa-se que a estação de vermelho apresentou o pior índice de qualidade isso se deve pelo uso e ocupação do solo na área ser predominante de urbanização, localizada na cidade de Cândido Sales.

\subsection{Aplicação da estatística multivariada}

Após aplicação da análise de Cluster para todos os parâmetros avaliados, nota-se que foram divididos em quatro grupos de similaridade (Figura 4). No primeiro grupo, os parâmetros de fósforo e DBO foram destacados dos demais, podendo ser explicados por serem os parâmetros que apresentaram os valores mais baixos em relação pela lei CONAMA n³57/2005.

O segundo grupo foi composto pelos parâmetros que ficaram acima do recomendado pela lei, sendo eles temperatura, condutividade elétrica, pH, nitrato, E. coli, DQO e coliformes totais. Tais parâmetros são característicos de efluentes domésticos e industriais, o que também foi encontrado por Nonato et al. (2007) em seu estudo de qualidade da água no rio das Velhas -MG. Os altos valores de condutividade podem indicar uma mineralização do processo que pode ser uma consequência da reciclagem de resíduos de atividades humanas desenvolvidas na área (Peluso et al., 2020).

Figura 4. Dendrograma da análise de Cluster para os parâmetros de qualidade da água.

\section{Dendograma de Cluster}

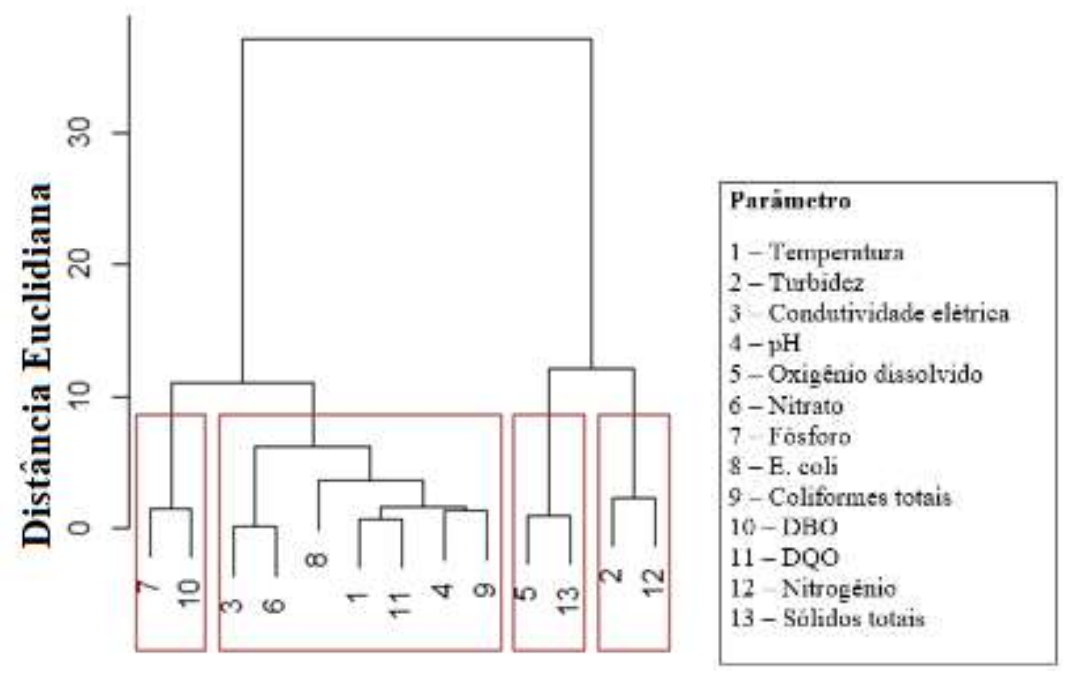

Fonte: Autores.

O terceiro grupo foi composto pelos parâmetros oxigênio dissolvido e sólidos totais. Nota-se que o oxigênio dissolvido foi um dos parâmetros que mais houve alterações em relação a lei o que pode estar associado a disponibilidade de nutrientes no meio. Contudo, Carrasco et al., (2019) encontrou resultado semelhante ao analisar a qualidade da água da bacia hidrográfica do Panamá.

Concluindo que, durante o verão, o influxo de nutrientes pode aumentar devido a fertilização das safras. Além disso, durante o inverno, as fortes chuvas causam aumento do escoamento de material orgânico, como folhas, troncos, vegetação e outros restos orgânicos que continuam a se decompor e, como resultado, dar origem a matéria orgânica e nutrientes.

O quarto grupo, foi composto pelo nitrogênio e turbidez no qual tais parâmetros ficaram de acordo com a legislação pertinente, não apresentando alterações na qualidade da água do rio. A principal causa da poluição por nitrogênio em ecossistemas aquáticos é atividades agrícolas (USTAOGLU, TEPE \& TAS, 2020). 
A segunda aplicação de Cluster (Figura 5) foi para analisar as estações em relação ao Índice de Qualidade de Água média na qual foram divididos em três grupos. O primeiro grupo (vermelho) foi composto pelas estações PD005 e PD007 sendo as estações mais críticas da bacia, apresentando os menores valores de IQA e caracterizada como a região mais poluída.

Figura 5. Dendrograma da análise de Cluster para as estações de monitoramento em relação aos dados de IQA.

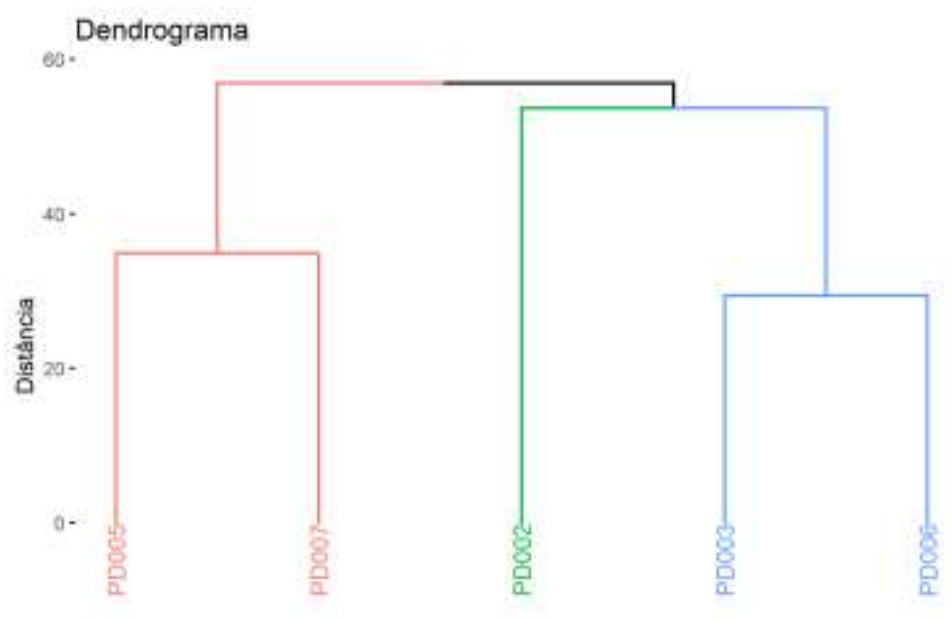

Fonte: Autores.

O segundo grupo (verde) foi composto pela estação PD002 caracterizada como moderadamente poluída, apresentando um valor médio de IQA. O terceiro grupo (azul) foi composto pelas estações PD003 e PD006 em que apresentaram o IQA mais alto das estações analisadas na bacia.

Os componentes principais (CP) gerados no programa Rstudio estão apresentados na Tabela 6, apresentando dois componentes principais que explicam no total $78,1 \%$ dos dados.

Tabela 6. Análise de Componentes Principais.

\begin{tabular}{|c|c|c|}
\hline PARÂMETROS & CP1 & CP2 \\
\hline$C T$ & $-0,03$ & $-0,15$ \\
\hline$P T$ & 0,13 & 0,02 \\
\hline Nitrato & $-0,09$ & $-0,001$ \\
\hline$N T$ & $-0,17$ & 0,36 \\
\hline$O D$ & $-0,03$ & 0,19 \\
\hline$p H$ & $-0,24$ & 0,24 \\
\hline$S T$ & 0,04 & $-0,05$ \\
\hline Temp & $-0,29$ & 0,33 \\
\hline Turb & $-0,58$ & $-0,21$ \\
\hline$D Q O$ & 0,38 & $-0,53$ \\
\hline$D B O$ & 0,13 & 0,02 \\
\hline Condut. Elétrica & 0,07 & $-0,01$ \\
\hline E. coli & $-0,53$ & $-0,56$ \\
\hline \% Variância Total & 51,35 & 26,75 \\
\hline \% Variância Acumulada & 51,35 & 78,1 \\
\hline
\end{tabular}

Fonte: Autores. 
De acordo com a Tabela 6, a CP1 explica 51,35\% da variância total dos dados, nos quais se destacaram os parâmetros de turbidez e E.Coli que foram diretamente proporcinais, podendo ser explicado pela presença de esgotos domésticos lançados no corpo hídrico. Segundo Cavalcante et al. (2017), a presença de E.coli pode ser indícios de esgotamento sanitário e de fezes animai. Além disso, Cavalcante et al.(2017) apresenta em seu trabalho que os parâmetros de turbidez e E.coli refletem alterações de natureza química, física e biológica da água, pois pode haver adsorçãode organismos patogênicos e nutrientes aos sólidos em suspensão.

A CP2 explica 26,75\% da variância total dos dados, em que os parâmetros que se destacaram foram DQO e E.coli. Segundo Santos et al. (2018) o parâmetro de DQO é responsável pela presença de matéria orgânica no corpo hídrico e a presença de E.coli é devido a descarga de esgoto doméstico no corpo hídrico.

\section{Considerações Finais}

A utilização de técnicas multivariadas para a avaliação dos dados de monitoramento de qualidade das águas superficiais da bacia hidrográfica do rio Pardo, buscou identificar os principais parâmetros responsáveis pela degradação da bacia. Dentre as técnicas, foram utilizadas a Análise de cluster e a Análise de Componentes Principais nas quais se mostraram eficientes na identificação das estações de monitoramento que apresentaram os menores IQA e dos parâmetros de qualidade da água mais relevantes para a caracterização da qualidade da água.

A análise cluster agrupou os parâmetros em quatro grupos e os índices de qualidade da água em três grupos, sendo o primeiro grupo como mais poluído contendo as estações PD005 e PD007 principalmente por esgoto doméstico, o segundo como moderadamente poluído com a estação PD002 e o terceiro grupo como o menos poluído com as estações PD003 e PD006. Em relação aos principais parâmetros responsáveis pela degradação da bacia, se destaca o parâmetro E.coli que em todas as estações da bacia apresentou violação crítica e se destacou como o principal componente principal, seguido da turbidez e da DQO. Portanto, pode-se ressaltar a importância de monitorar de forma homogênea os parâmetros da qualidade da água em todas as estações de monitoramento por meio do IQA ligado as técnicas de estatísticas multivariadas. Ademais, como possibilidade de estudos futuros, avaliar outros parâmetros de qualidade de água, dentre as quais: alcalinidade e metais pesados podem ser estudados para um melhor entendimento da poluição pontual e difusa que ocorrem nesta bacia. Além disso, outras técnicas estatísticas multivariadas como análise discriminante e análise fatorial podem ser analisadas para um estudo mais aprofundado da qualidade da água nesta bacia.

\section{Agradecimentos}

O presente trabalho foi realizado com apoio da Coordenação de Aperfeiçoamento de Pessoal de Nível Superior Brasil (CAPES).

\section{Referências}

Ana - Agência Nacional de Águas. (2009) Indicadores de Qualidade- indice de qualidade das águas. <http://pnqa.ana.gov.br/indicadoresQA/IndiceQA.aspx>.

Anteneh et al. (2018). Assessment of surface water quality in Legedadie and Dire catchments, Central Ethiopia, using multivariate statistical analysis. Acta Ecologica Sinica. 38 < https://doi.org/10.1016/j.chnaes.2017.05.005>

Bodrud-Doza et al. (2016) Characterization of groundwater quality using water evaluation indices, multivariate statistics and geostatistics in central Bangladesh. Water Science. $30<$ https://doi.org/10.1016/j.wsj.2016.05.001>

Carrasco, G. et al. (2019). Water quality evaluation through a multivariate statistical HJ-Biplot approach. Journal of Hydrology. 577. < https://doi.org/10.1016/j.jhydrol.2019.123993>. 
Castro Junior, R. M.; Sobreira, F. G.; \& Bortoloti, F. D. (2007). Modelagem geoestatística a partir de parâmetros da qualidade da água (IQA-NSF) para a subbacia hidrográfica do rio Castelo (ES) usando sistema de informações geográficas. Revista Brasileira de Cartografia. 59(3).

Cavalcante, I., Silva, R. S., Blanco, C. J. C., Teixeira, L. C. G. M., \& Fernandes, L. L (2017). Análise da qualidade da água de uma bacia hidrográfica periurbana no município de Capitão Poço/PA. Revista AIDIS, 12, 413-431.

Cecconella, S. T., Centeno, L. N., \& Guedes, H. A. S. (2018) Índice de qualidade de água modificado pela análise multivariada: estudo de caso do Arroio Pelotas, RS, Brasil. Engenharia Sanitária e Ambiental. 23(5), 973-978. https://doi.org/10.1590/s1413-41522018165394

CONAMA (2005) - Conselho Nacional do Meio Ambiente. Resolução $n^{o}$ 357, de 17 de março de 2005. Dispõe sobre a classificação dos corpos de água e diretrizes ambientais para o seu enquadramento, bem como estabelece as condições e padrões de lançamento de efluentes, e dá outras providências. Diário Oficial da União, Brasília, DF.

Li et al. (2018). A comparative assessment of Australia's Lower Lakes water quality under extreme drought and post-drought conditions using multivariate statistical techniques. Journal of Cleaner Production. 190. https://doi.org/10.1016/j.jclepro.2018.04.121

Liu, S., Ryu, D., Webb, J. A., Lintern, A., Waters, D., Guo, D., \& Western, A. W. (2018). Characterisation of spatial variability in water quality in the Great Barrier Reef catchments using multivariate statistical analysis. Marine Pollution Bulletin, 137(October), 137-151. https://doi.org/10.1016/j.marpolbul.2018.10.019.

Lopes, F. B. et al. (2008). Mapa da qualidade das águas do rio Acaraú, pelo emprego do IQA e Geoprocessamento. Revista Ciência Agronômica. 39(3).

Maia, K. P., Silva, G. A., \& Libânio, M. (2019) Aplicação de análise multivariada no estudo da frequência de amostragem e do número de estações de monitoramento de qualidade da água. Engenharia Sanitária e Ambiental, 24(5). https://doi.org/10.1590/s1413-41522019175743

Miyittah et al. (2020). Assessment of surface water quality status of the Aby Lagoon System in the Western Region of Ghana. Heliyon. 6 < https://doi.org/10.1016/j.heliyon.2020.e04466>

Muangthong, S., \& Shrestha, S. (2015). Assessment of surface water quality using multivariate statistical techniques: case study of the Nampong River and Songkhram River, Thailand. Environmental Monitoring and Assessment, 187(9). <https://doi.org/10.1007/s10661-015-4774-1>

Nonato et al. (2007) Tratamento estatístico dos parâmetros da qualidade das águas da bacia do alto curso do Rio das Velhas. Química Nova. 30. < http://dx.doi.org/10.1590/S0100-40422007000400008>

NSF - National Sanitation Foundation (2007). Water Quality Index. 〈http://www.nsf.org/consumer/earth_day/wqi.asp>

Oliveira, S. L. (2017) Avaliação da qualidade da água do riacho São Caetano, de Balsas (MA), com base em parâmetros físicos, químicos e microbiológicos. Revista Engenharia Sanitária e Ambiental. 22 (3) 523-529.

Peluso et al. (2020). Integrated analysis of the quality of water bodies from the lower Parana River basin with different productive uses by physicochemical and biological indicators. Environmental Pollution. $263<$ https://doi.org/10.1016/j.envpol.2020.114434>

Santos, S. A. et al. (2018). Qualidade da água na bacia hidrográfica urbana Cancela Tamandaí, Santa Maria/RS. Soc. Nat., 30(2), 23-44 < I: http://dx.doi.org/10.14393/SN-v30n2-2018-2-X>

Seu-Anoï et al., (2018). Distribuição espacial e temporal de cianobactérias e sua relação com parâmetros ambientais no Sistema Lagunar de Aby (sudeste da Costa do Marfim, África Ocidental). Aust. J. Basic Appl. Sci., 12 (1), 37 - 44.

Unda-Calvo, J. et al. (2019). Antigüedad, Multivariate statistical analyses for water and sediment quality index development: a study of susceptibility in na urban river, Science of the Total Environment. https://doi.org/10.1016/j.scitotenv.2019.135026

Ustaoglu, T. T. (2020). Assessment of stream quality and health risk in a subtropical Turkey river system: A combined approach using statistical analysis and water quality index. Ecological Indicators. $113<$ https://doi.org/10.1016/j.ecolind.2019.105815>

Von Sperling, M. (2007) Estudos e modelagem da qualidade da água de rios. Princípios do tratamento biológico de águas residuárias. (3a ed.), Departamento de Engenharia Sanitária e Ambiental; Universidade Federal de Minas Gerais, 7. 